\title{
Mutual Coupling Reduction between Patch Antennas Using Meander Line
}

\author{
Qian Li D, ${ }^{1}$ Chong Ding, ${ }^{1}$ Ruichao Yang, ${ }^{1}$ Mingtao Tan, ${ }^{2}$ Gangxiong Wu, ${ }^{1}$ Xia Lei, \\ Xuebing Jiang, ${ }^{1}$ Shuanzhu Fang, ${ }^{1}$ Minzhi Huang, ${ }^{1}$ Yubin Gong, ${ }^{1}$ and Yanyu Wei ${ }^{1}$ \\ ${ }^{1}$ School of Electronic Science and Engineering, University of Electronic Science and Technology of China, Chengdu 610054, China \\ ${ }^{2}$ School of Electrical and Information Engineering, Hunan University of Arts and Science, Changde 415000, China \\ Correspondence should be addressed to Qian Li; qianli_uestc@163.com
}

Received 5 February 2018; Accepted 22 May 2018; Published 4 July 2018

Academic Editor: Muhammad Ramlee Kamarudin

Copyright (C) 2018 Qian Li et al. This is an open access article distributed under the Creative Commons Attribution License, which permits unrestricted use, distribution, and reproduction in any medium, provided the original work is properly cited.

\begin{abstract}
Meander lines (MLs) in two configurations are presented to reduce the mutual coupling (MC) between two microstrip patch antenna elements. Inserting a slot in the ground plane between the antenna elements is a simple method to reduce the MC, while adding the MLs in the slot of the ground can further reduce the MC. In the first configuration, one ML is inserted in the slot of the ground and a maximum MC reduction of $39 \mathrm{~dB}$ throughout the $-10 \mathrm{~dB}$ bandwidth is achieved. What's more, the radiation patterns are not changed compared with the dual-element microstrip antenna array with a slotted ground. For the second configuration, two MLs are added in the slot of the ground. It is found that a maximum isolation of $53 \mathrm{~dB}$ can be obtained. However, the radiation patterns are slightly changed compared with the dual-element microstrip antenna array with a slot in the ground. Meanwhile, the measured peak gain and efficiency of the dual-element microstrip antenna array in the two configurations are given. Along with this paper, several prototypes have been fabricated and measured. The simulated results are in good accordance with the measurements, which are presented to verify that MC reduction can be achieved between microstrip antenna elements by adding the MLs in the slotted ground.
\end{abstract}

\section{Introduction}

Microstrip patch antennas are widely used in a lot of applications, such as multiple input and multiple output (MIMO) communication systems. The main advantages of microstrip patch antennas are their low profile, low cost, and ease of manufacture [1-4]. However, microstrip patch antennas exhibit MC when used as array elements [5]. Mutual coupling has a direct impact on the performance of multiantenna systems. High MC can degrade a system's performance such as lower antenna efficiency, impedance mismatching, and deviation of the radiation pattern [6]. Thus, reducing mutual coupling is important in multiantenna systems.

To deal with the problem of MC, the direct solution is to enlarge the space between the microstrip antenna elements. It is recommended that the distance between the neighboring antenna elements has to be larger than $0.5 \lambda$ [7], which is contrary to compact designs. The reduction of mutual coupling has drawn wide attention in the antenna community. Several methods have been suggested in the literature to reduce MC while keeping the antenna elements close to each other, including the use of electromagnetic bandgap structures $[8,9]$, defective ground structures $[1,10-12]$, metamaterials $[6,13,14]$, resonators [15], and slots $[16,17]$.

In this paper, we present two schemes that utilize the MLs to reduce the MC between two microstrip antenna elements. The MLs in two configurations are placed in the slotted ground plane with a separation of $1 / 5$ free-space wavelength, which is equal to the edge-to-edge distance of the antenna elements. In the first configuration, one ML is inserted in the slot of the ground, which creates an additional coupling path to compensate for the existing electromagnetic coupling. For the second configuration, two MLs are added in the slot of the ground, which introduces two coupling paths. A signal coming via one of the two coupling paths opposes the signal going from the other coupling path, which leads 


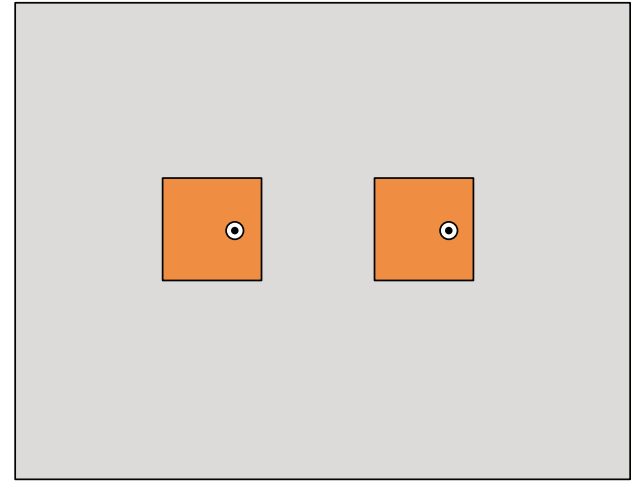

(a)

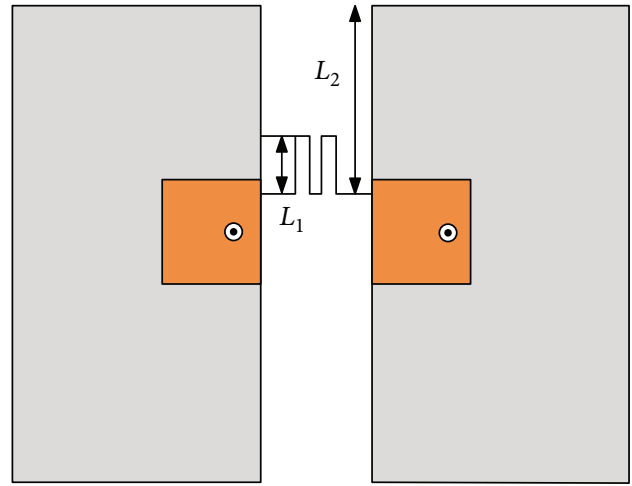

(c)

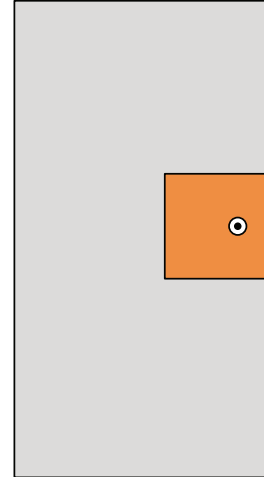

(b)
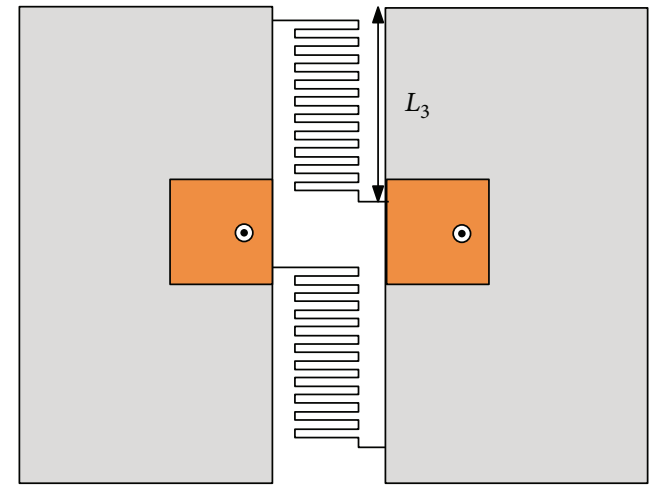

(d)

Figure 1: The design evolution of the dual-element microstrip antenna array equipped with MLs. (a) Prototype A. (b) Prototype B. (c) Prototype C. (d) Prototype D.

to a reduction of MC. By using the MLs in the slotted ground, a significant reduction of MC between two microstrip antenna elements can be obtained. Meanwhile, the radiation patterns in the first configuration are not changed compared with the dual-element microstrip array with a slotted ground. However, compared to the dual-element microstrip array with the slotted ground, the radiation patterns in the second configuration are slightly changed. In Section 2, the antenna design is performed and the mechanism of the MLs in two configurations employed in the dual-element microstrip antenna array is described. Then, we further investigate the effect of the important parameters on the MC reduction. In Section 3, the comparisons between experimental and simulated results are presented, including the $S$ parameters and radiation patterns. Finally, a conclusion of the proposed scheme is given in Section 4.

\section{Antenna Configuration and Design Consideration}

Since the MLs in two configurations are used to reduce the MC, a conventional dual-element microstrip antenna array has been chosen to demonstrate the capability of the MLs. The geometry of the conventional dual-element microstrip antenna array is shown in Figure 1(a). The two identical microstrip patch antennas have an area of $13.2 \times 14 \mathrm{~mm}^{2}$ with the overall board size of $78 \times 60 \mathrm{~mm}^{2}$. They are printed on the top side of the FR4 substrate with a thickness of
$1.6 \mathrm{~mm}$ and a dielectric constant of 4.4. Meanwhile, the two patch antenna elements resonating at $5 \mathrm{GHz}$ are placed 0.2 $\lambda_{0}$ apart, where $\lambda_{0}$ is the free-space wavelength.

MC between patch elements can be attributed to nearfield coupling, far-field coupling, and surface-wave coupling. The near-field coupling occurs when one antenna is in the near-field zone of another antenna, the far-field coupling occurs due to the patch radiation in horizontal directions, and the surface-wave coupling is dominant when the normalized substrate electrical thickness $h / \lambda_{0}$ is on the order of (1) or larger [18]:

$$
\frac{h}{\lambda_{0}}=\frac{3}{2 \pi \sqrt{\varepsilon_{r}}},
$$

where $h$ is the substrate thickness, $\lambda_{0}$ is the free-space wavelength, and $\varepsilon_{r}$ is the relative permittivity of the substrate. From (1), we can see that the dominant coupling between the microstrip antenna elements chosen in this paper is surface-wave coupling. The slot inserting in the ground is a simple and popular method to reduce MC between the microstrip antennas, but the effect on the surface-wave coupling reduction is not favorable. The MLs as extra coupling paths in two configurations are inserted in the slot of the ground in order to further reduce the surface-wave coupling between the patch elements.

The design evolution of the dual-element microstrip antenna array equipped with MLs are presented in Figure 1. 


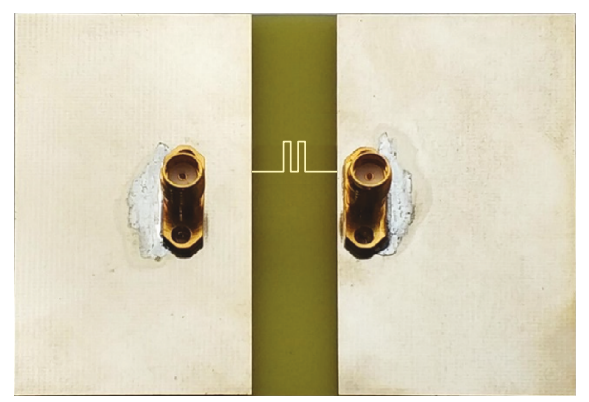

(a)

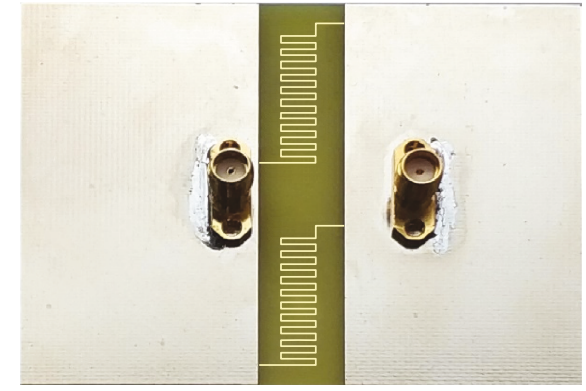

(b)

Figure 2: The photograph of the dual-element microstrip antenna array equipped with MLs. (a) The first configuration. (b) The second configuration.

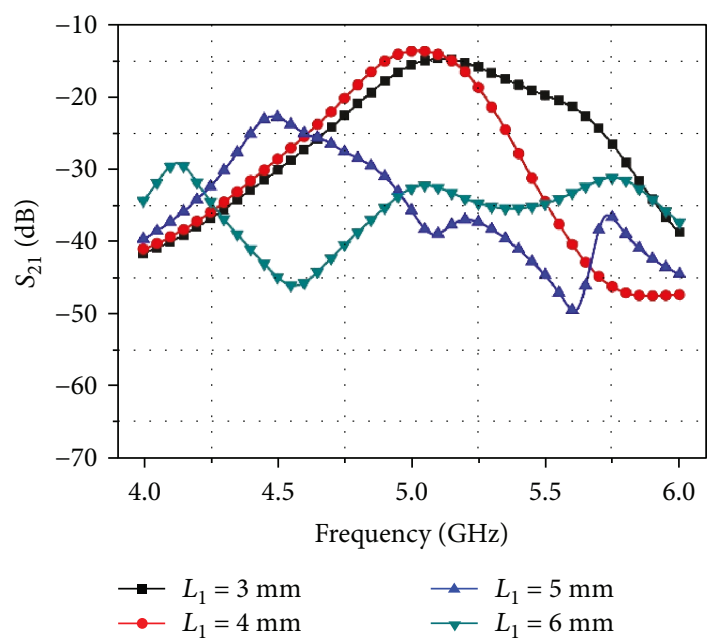

(a)

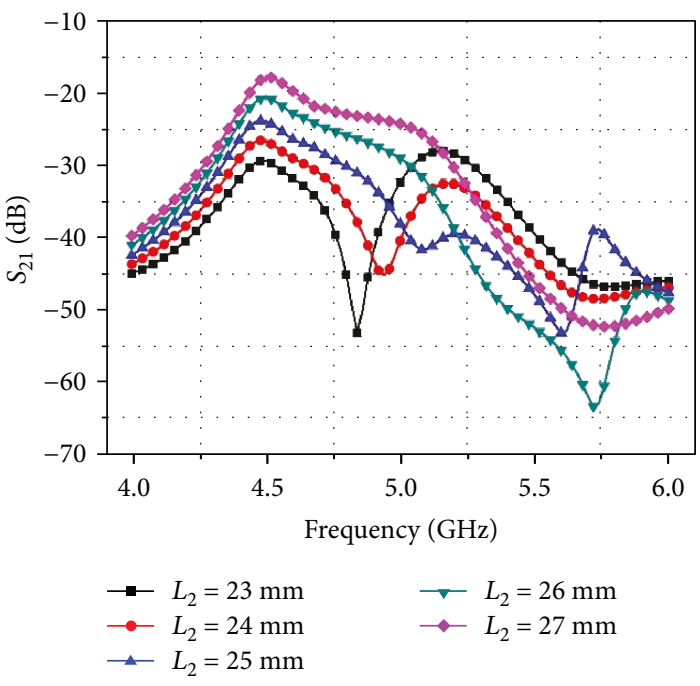

(b)

FIGURE 3: (a) Simulated $S_{21}$ varying with the parameter $L_{1}$. (b) Simulated $S_{21}$ varying with different $L_{2}$.

Prototype A is a conventional dual-element microstrip antenna array. The dual-element microstrip antenna array with a slot in the ground between the antennas is denoted as prototype $B$. The width of the slot is equal to the distance between the elements. Prototype $\mathrm{C}$ refers to the dual-element antenna array loaded with one ML for the first configuration. Prototype D denotes the dual-element antenna array equipped with two MLs for the second configuration. The gray shaded area represents the ground plane of the microstrip antenna array.

The prototypes A, B, C, and D have been fabricated and measured. Figure 2 shows a photograph of the dual-element microstrip antenna array equipped with MLs in two configurations. The mechanism of the MLs in two configurations employed in the dual-element microstrip antenna array will be described below.

2.1. The First Configuration. The surface-wave coupling between two elements can be canceled out by properly adding an extra coupling path. The design aims at creating an extra coupling that opposes the signal going from one antenna element to the other. After several trials, we succeeded in inserting an ML in the proper position on the slot in the ground. The optimized structure is presented in Figure $1(\mathrm{c})$. The width of the metal strip is $0.2 \mathrm{~mm}$. The total length of the metal strip $L$ is $32 \mathrm{~mm}$. The transverse length of the metal strip $L_{1}$ is $5 \mathrm{~mm}$. The thickness of the metal strip $t$ is $0.017 \mathrm{~mm}$. The distance from the loading position to the top of the substrate $L_{2}$ is $25 \mathrm{~mm}$.

Several parameters have an effect on MC, and some key parameters are studied below. When one parameter is studied, the others are kept constant. The transverse length of the metal strip $L_{1}$ and the distance from the loading position to the top of the substrate $L_{2}$ are important in reducing the MC between the two antenna elements. In order to investigate the effects of $L_{1}$ on the MC reduction, we have performed parametric studies as shown in Figure 3(a). From Figure 3(a), we can see that the isolation between the dualelement microstrip antenna array with the first configuration is sensitive with $L_{1}$. It is evident that when $L_{1}$ is equal to $5 \mathrm{~mm}$, a maximum isolation of $39 \mathrm{~dB}$ can be achieved within the $-10 \mathrm{~dB}$ impedance bandwidth. Meanwhile, the effect of the length $L_{2}$ on $S_{21}$ is analyzed as shown in Figure 3(b). The isolation is also sensitive with this parameter. It is observed that as $L_{2}$ varies from 23 to $27 \mathrm{~mm}$, the maximum isolation appears when $L_{2}$ is equal to $25 \mathrm{~mm}$. Therefore, 


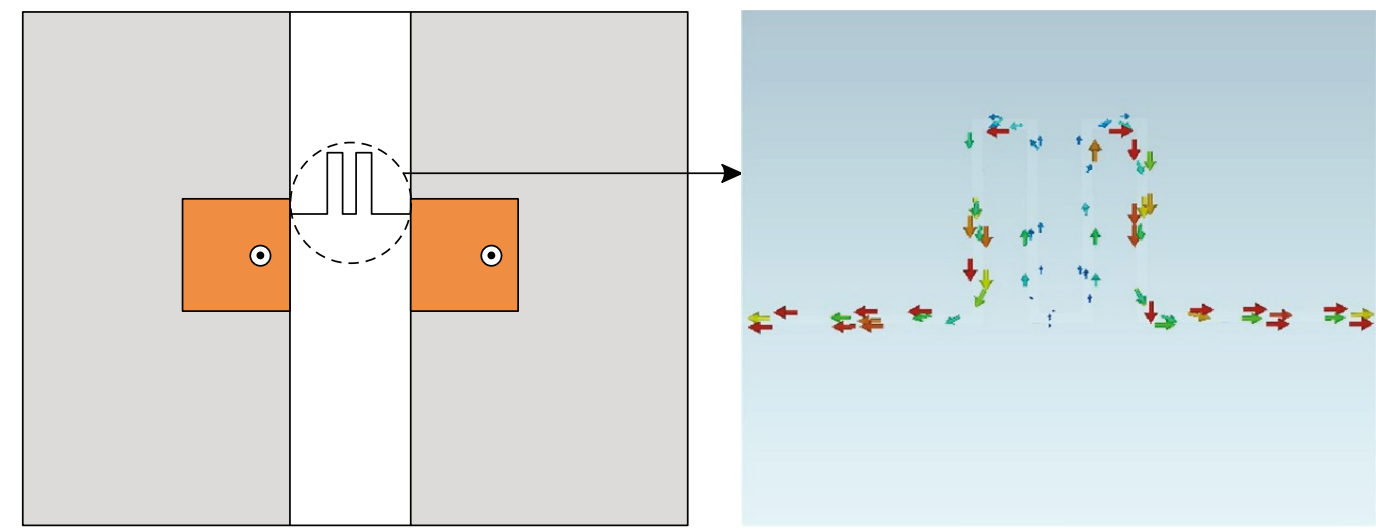

Figure 4: The antenna array with the ML in the first configuration and the current distribution of the ML.

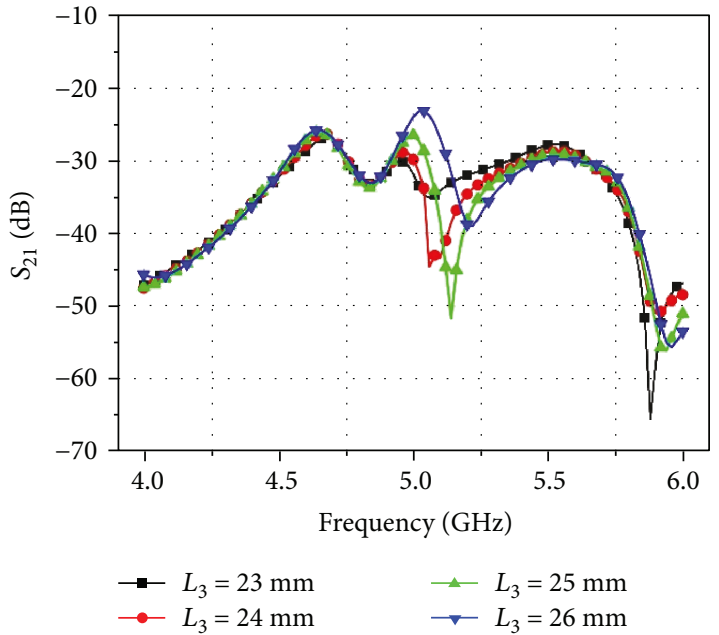

(a)

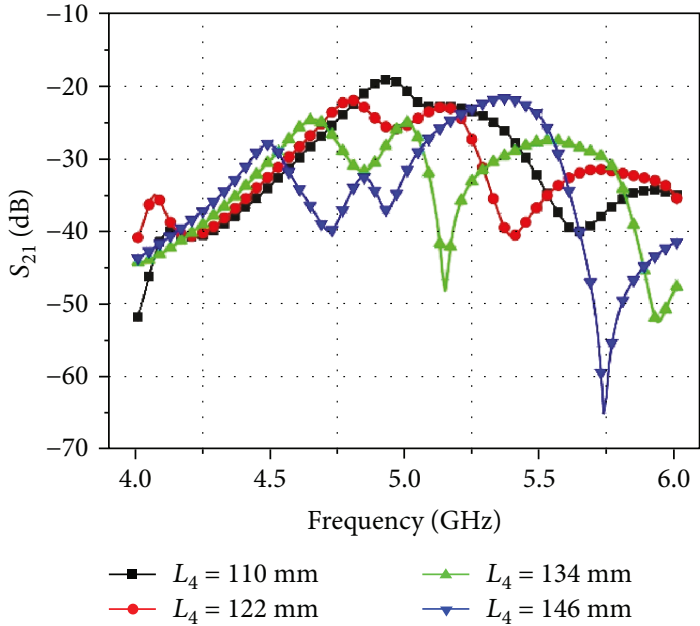

(b)

Figure 5: (a) Simulated $S_{21}$ for different $L_{3}$. (b) Simulated $S_{21}$ for different $L_{4}$.

through optimization of this parameter, $L_{2}=25 \mathrm{~mm}$ is chosen for good isolation.

What's more, realizing mutual coupling reduction while keeping the radiation patterns is very important. In the first configuration, by introducing an extra coupling path, the mutual coupling is considerably reduced compared with the dual-element microstrip antenna array with slotted ground. Figure 4 shows the antenna array with the ML in the first configuration and the current distribution of the ML. From the current distribution of the ML as shown in Figure 4, we can see that the currents flowing on the ML are opposite, which causes their contribution to the far field to be canceled by each other. Thus, the radiation patterns of the design are almost not changed compared with the dual-element microstrip antenna array with slotted ground.

2.2. The Second Configuration. By adding two MLs in the slot of the ground, two coupling paths are created. The design aims at creating two signals: one signal coming via one of the coupling path opposes the other signal going via the other coupling path. If the amplitudes of the two signals are comparable, the mutual coupling can be reduced considerably.
The optimized structure is presented in Figure 1(d). The parameters of the two MLs are the same: the width of the metal strip is $0.2 \mathrm{~mm}$; the distance from the loading position to the top of the substrate $L_{3}$ is $25 \mathrm{~mm}$; the total length of the metal strip $L_{4}$ is $134 \mathrm{~mm}$; and the thickness of the metal strip tis $0.017 \mathrm{~mm}$.

The important parameters are invested below. When one parameter is studied, the others are kept constant. Analyses of the effect of $L_{3}$ and $L_{4}$ on $S_{21}$ are plotted in Figure 5 . As can be seen from the Figure $5(\mathrm{a}), L_{3}$ significantly influences $S_{21}$, and good reduction in mutual coupling can be achieved when $L_{3}$ is equal to $25 \mathrm{~mm}$. Figure 5(b) shows the simulated $S_{21}$ for various $L_{4}$. It is observed that when $L_{4}$ is equal to $134 \mathrm{~mm}$, the maximum isolation appears at resonant frequency.

In the second configuration, two extra coupling paths are introduced and the mutual coupling is considerably reduced compared with the dual-element microstrip antenna array with slotted ground. In order to clearly show the current distribution of the two MLs, part of the current distribution is given which is shown in Figure 6. From Figure 6, we can see that the current on the two MLs are not absolutely 


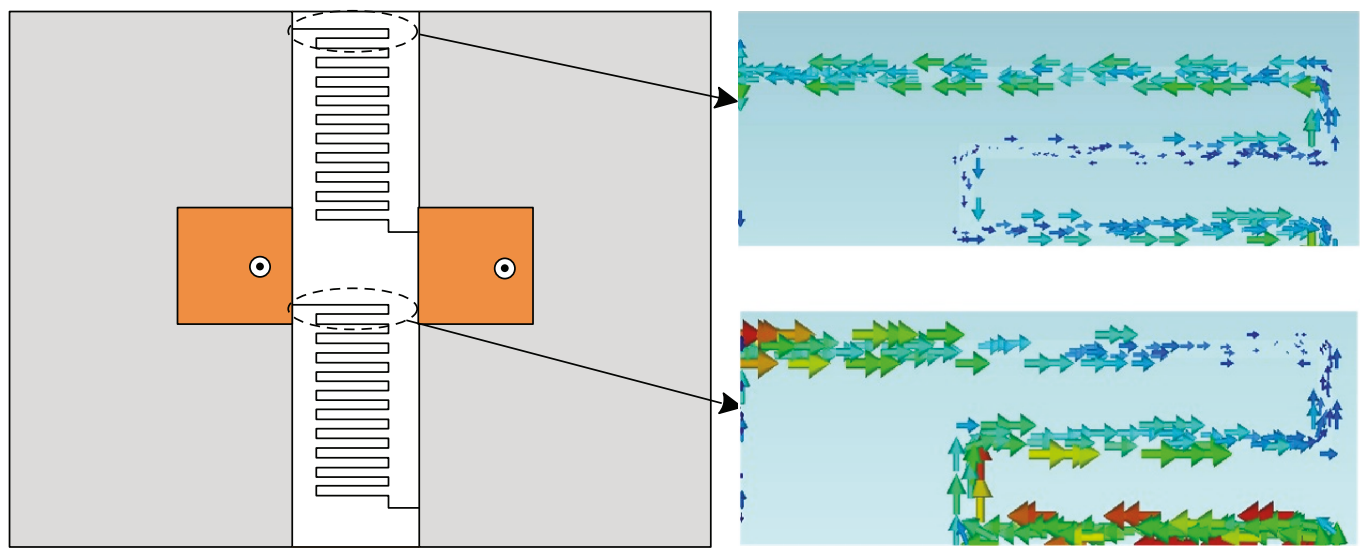

Figure 6: The antenna array with the ML in the second configuration and the simulated current density vectors on the ML.

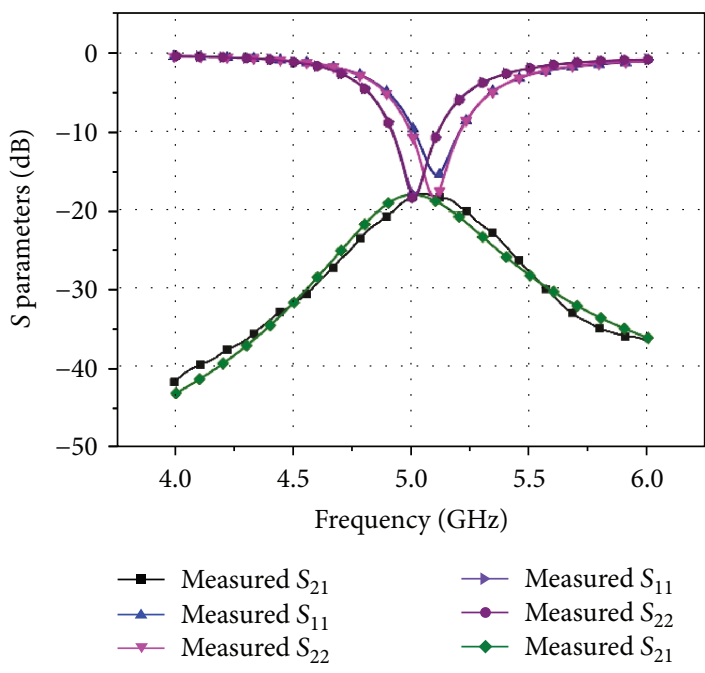

(a)

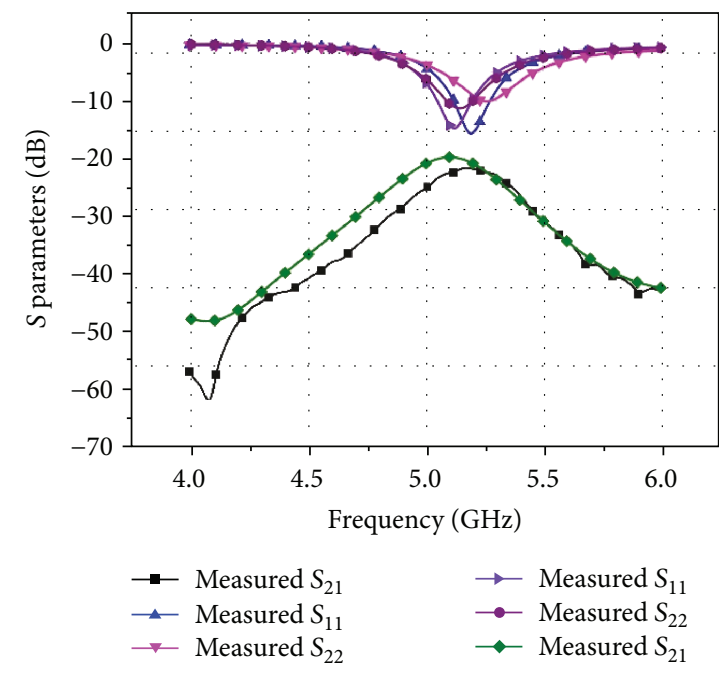

(b)

FIGURE 7: (a) Simulated and measured $S$ parameters of prototype A. (b) Simulated and measured $S$ parameters of prototype B.

canceled, which cannot prevent influence on the radiation patterns of the antenna elements. So the radiation patterns are slightly changed.

\section{Experimental Results and Discussions}

In order to verify the effectiveness of the dual-element microstrip antenna array loaded with MLs on the MC reduction, the prototypes $\mathrm{A}, \mathrm{B}, \mathrm{C}$, and $\mathrm{D}$ have been fabricated and measured.

Figure 7(a) shows the comparisons of the simulated and measured $S$ parameters of the conventional microstrip antenna array (namely prototype A), which indicate that the measured results have a slight shift in the resonant frequency compared with the simulated ones. The simulated and measured $S$ parameters of the microstrip antenna array with a slotted ground plane (namely prototype B) are shown in Figure 7(b). As can be seen, the measured results are a little smaller compared with the simulated ones, and a slight shift in the resonant frequency occurred. Figure 8(a) shows the comparisons of the simulated and measured $S$ parameters of the proposed antenna $\mathrm{C}$, which indicate that the measured results agree well with the simulated ones except for a slight shift in the resonant frequency. The slight discrepancy may be caused by fabrication error and measurement error. Figure $8(\mathrm{~b})$ shows the comparisons of the simulated and measured $S$ parameters of the proposed antenna $\mathrm{D}$, which indicate that the measured $S$ parameters are in accordance with the simulated ones. From Figures 7 and 8, we can see that the microstrip antenna array with a slotted ground plane provides an improvement of isolation of about $4 \mathrm{~dB}$ more than the conventional microstrip antenna array, and the slot in the ground affects the resonant frequency a little. By adding the ML in the first configuration in the slot of the ground (namely prototype $\mathrm{C}$ ), the isolation is improved by about $18 \mathrm{~dB}$ compared with prototype B. Meanwhile, by inserting two MLs in the slotted ground (namely prototype D), an additional $31 \mathrm{~dB}$ of mutual coupling is reduced at a resonant frequency compared with prototype $B$.

The far-field radiation patterns of the four dual-element microstrip antenna arrays are measured and compared. The radiation patterns are measured by exciting one of the 


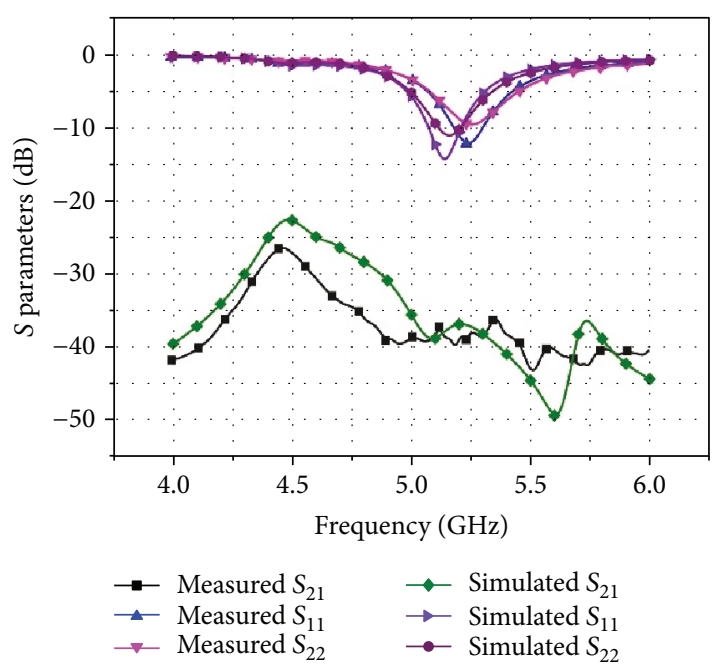

(a)

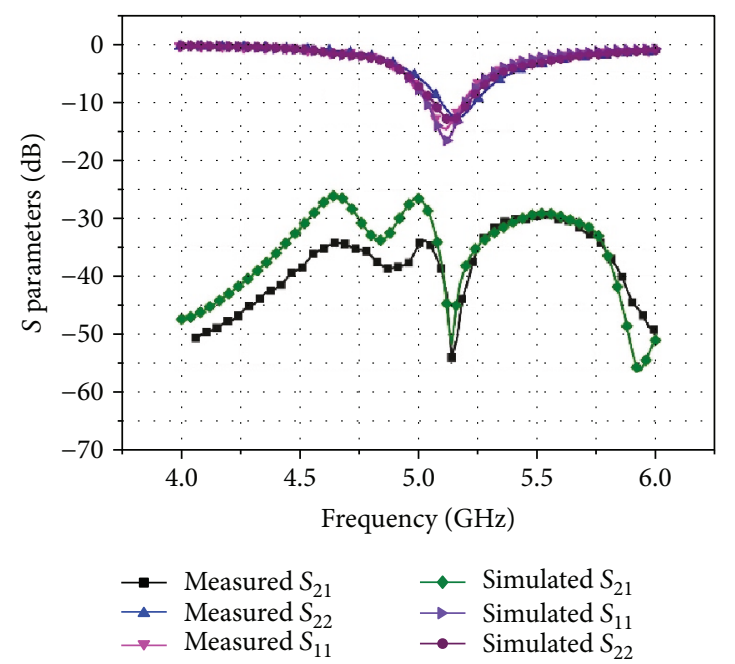

(b)

FIGURE 8: (a) Simulated and measured $S$ parameters of prototype C. (b) Simulated and measured $S$ parameters of prototype D.

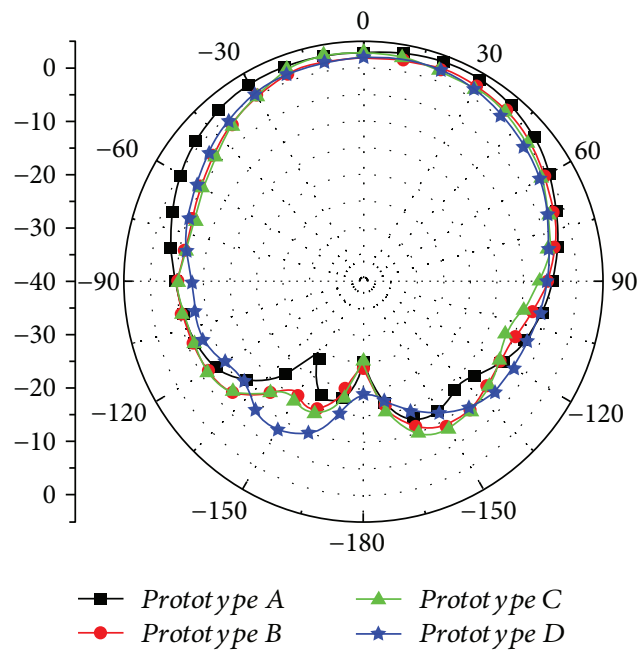

(a)

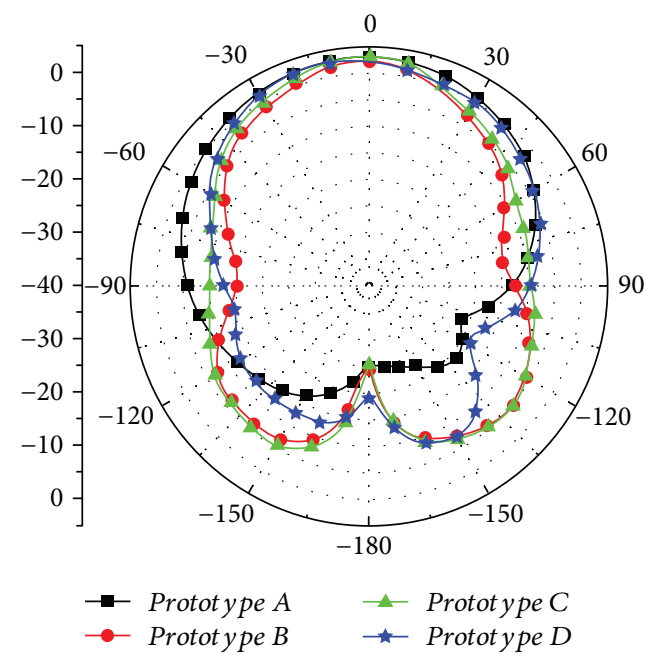

(b)

FIGURE 9: Far-field radiation patterns of the three antennas in (a) xoz plane and the (b) yoz plane.

antennas and terminating the other with a $50 \Omega$ load. Figure 9 shows the measured radiation patterns of prototypes $\mathrm{A}, \mathrm{B}, \mathrm{C}$, and $\mathrm{D}$ in the xoz plane and yoz plane. The results show that the back radiation of prototype $\mathrm{B}$, prototype $\mathrm{C}$, and prototype $\mathrm{D}$ are stronger than that of prototype $\mathrm{A}$, while the forward radiation has little difference, which is because the slot in the ground plane can force the power coupling from one antenna element to the other to radiate into space. The radiation patterns of prototype $\mathrm{C}$ are almost the same with prototype $\mathrm{B}$, which demonstrates that the loaded ML in the first configuration does not change the radiation characteristics. However, the radiation patterns of prototype D are slightly changed.

Meanwhile, the measured peak gain and efficiency of prototype $\mathrm{C}$ and prototype $\mathrm{D}$ are plotted in Figure 10. It is seen that the measured peak gain of prototype $\mathrm{C}$ is $3.42 \mathrm{~dB}$ and that of prototype $\mathrm{D}$ is $4.04 \mathrm{~dB}$, and the corresponding maximum efficiency is $41.47 \%$ and $51.51 \%$, respectively.

\section{Conclusions}

Meander lines (MLs) in two configurations have been proposed to reduce the MC between two coplanar microstrip antenna elements. Inserting a slot in the ground achieves an improvement of $4 \mathrm{~dB}$ compared with the conventional dual-element microstrip antenna array. By adding MLs in two configurations in the slot in the ground, a reduction in MC of more than $17 \mathrm{~dB}$ and $31 \mathrm{~dB}$ between two coplanar microstrip antenna elements are obtained, respectively. The radiation patterns of the dual-element microstrip antenna array with a slotted ground and the dual-element microstrip antenna array loaded with an ML in the first configuration are almost the same. That means the loading of the ML in the first configuration in the slot can effectively improve the isolation and does not change the radiation patterns. However, the dual-element microstrip antenna array loaded with an $M L$ in the second configuration has a better effect on the 


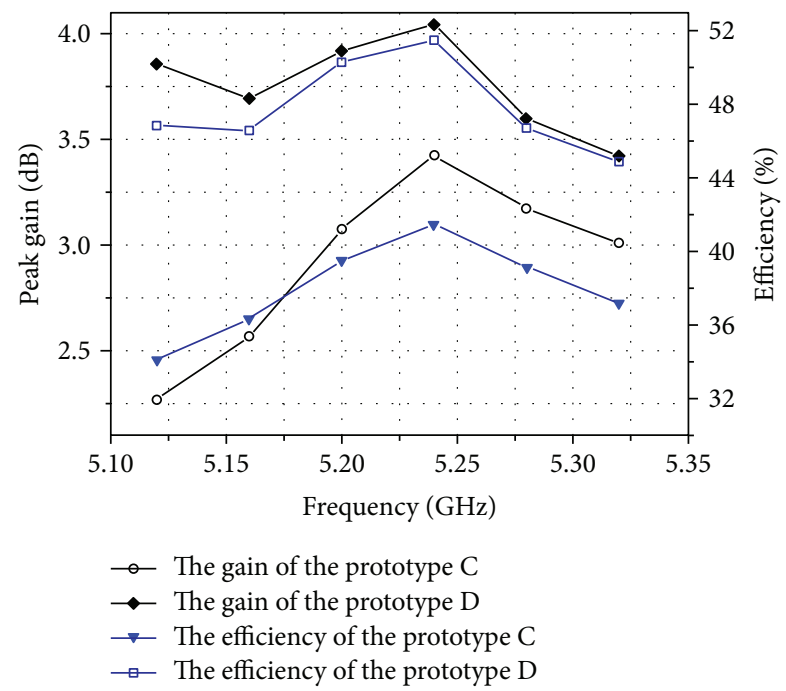

FIgURE 10: The measured peak gain and efficiency of prototype C and prototype $\mathrm{D}$.

MC reduction while the radiation patterns are slightly changed. Moreover, the measured peak gain and efficiency of the dual-element microstrip antenna array loaded with an ML in the first configuration is $3.42 \mathrm{~dB}$ and $41.47 \%$, respectively. For the second configuration, the corresponding peak gain and efficiency is $4.04 \mathrm{~dB}$ and $51.51 \%$, respectively. The technique proposed in this paper may be useful in a number of multiantenna systems such as MIMO antennas and arrays.

\section{Data Availability}

The data used to support the findings of this study are available from the corresponding author upon request.

\section{Conflicts of Interest}

The authors declare that they have no conflicts of interest.

\section{Acknowledgments}

This work was supported in part by the National Natural Science Foundation of China under Grant nos. 61771117, 61531010 , and 61271029.

\section{References}

[1] K. Wei, J. Y. Li, L. Wang, Z. J. Xing, and R. Xu, "Mutual coupling reduction by novel fractal defected ground structure bandgap filter," IEEE Transactions on Antennas and Propagation, vol. 64, no. 10, pp. 4328-4335, 2016.

[2] S. Hwangbo, H. Y. Yang, and Y. K. Yoon, "Mutual coupling reduction using micromachined complementary meanderline slots for a patch array antenna," IEEE Antennas and Wireless Propagation Letters, vol. 16, pp. 1667-1670, 2017.

[3] A. Emadeddin, S. Shad, Z. Rahimian, and H. R. Hassani, "High mutual coupling reduction between microstrip patch antennas using novel structure," AEU - International Journal of Electronics and Communications, vol. 71, pp. 152-156, 2017.
[4] G.-C. Wu, G.-M. Wang, J.-G. Liang, X.-J. Gao, and L. Zhu, "Novel ultra-compact two-dimensional waveguide-based metasurface for electromagnetic coupling reduction of microstrip antenna array," International Journal of RF and Microwave Computer-Aided Engineering, vol. 25, no. 9, pp. 789-794, 2015.

[5] R. D. Murch and K. B. Letaief, "Antenna systems for broadband wireless access," IEEE Communications Magazine, vol. 40, no. 4, pp. 76-83, 2002.

[6] X. M. Yang, X. G. Liu, X. Y. Zhou, and T. J. Cui, "Reduction of mutual coupling between closely packed patch antennas using waveguided metamaterials," IEEE Antennas and Wireless Propagation Letters, vol. 11, pp. 389-391, 2012.

[7] A. H. Mohammadian, N. M. Martin, and D. W. Griffin, "A theoretical and experimental study of mutual coupling in microstrip antenna arrays," IEEE Transactions on Antennas and Propagation, vol. 37, no. 10, pp. 1217-1223, 1989.

[8] E. Rajo-Iglesias, Ó. Quevedo-Teruel, and L. Inclan-Sanchez, "Mutual coupling reduction in patch antenna arrays by using a planar EBG structure and a multilayer dielectric substrate," IEEE Transactions on Antennas and Propagation, vol. 56, no. 6, pp. 1648-1655, 2008.

[9] J. Y. Lee, S. H. Kim, and J. H. Jang, "Reduction of mutual coupling in planar multiple antenna by using 1-D EBG and SRR structures," IEEE Transactions on Antennas and Propagation, vol. 63, no. 9, pp. 4194-4198, 2015.

[10] F. G. Zhu, J. D. Xu, and Q. Xu, "Reduction of mutual coupling between closely-packed antenna elements using defected ground structure," Electronics Letters, vol. 45, no. 12, pp. 601602, 2009.

[11] M. Salehi and A. Tavakoli, "A novel low mutual coupling microstrip antenna array design using defected ground structure," AEU - International Journal of Electronics and Communications, vol. 60, no. 10, pp. 718-723, 2006.

[12] A. Mahmoudian, J. Rashed-Mohassel, and J. A. Kong, "Reduction of EMI and mutual coupling in array antennas by using DGS and AMC structure," in Piers 2008 Hangzhou: Progress In Electromagnetics Research Symposium, Vols I and II, Proceedings, Electromagnetics Academy, p. 106, 2008.

[13] M. M. Bait-Suwailam, O. F. Siddiqui, and O. M. Ramahi, "Mutual coupling reduction between microstrip patch antennas using slotted-complementary split-ring resonators," IEEE Antennas and Wireless Propagation Letters, vol. 9, pp. 876$878,2010$.

[14] Z. Qamar and H.-C. Park, "Compact waveguided metamaterials for suppression of mutual coupling in microstrip array," Progress in Electromagnetics Research, vol. 149, pp. 183-192, 2014.

[15] J. Ghosh, S. Ghosal, D. Mitra, and S. R. Bhadra Chaudhuri, "Mutual coupling reduction between closely placed microstrip patch antenna using meander line resonator," Progress In Electromagnetics Research Letters, vol. 59, pp. 115-122, 2016.

[16] J. OuYang, F. Yang, and Z. M. Wang, "Reducing mutual coupling of closely spaced microstrip MIMO antennas for WLAN application," IEEE Antennas and Wireless Propagation Letters, vol. 10, pp. 310-313, 2011.

[17] M. Sonkki and E. Salonen, "Low mutual coupling between monopole antennas by using two $\lambda / 2$ slots," IEEE Antennas and Wireless Propagation Letters, vol. 9, pp. 138-141, 2010.

[18] M. M. Nikolic, A. R. Djordjevic, and A. Nehorai, "Microstrip antennas with suppressed radiation in horizontal directions and reduced coupling," IEEE Transactions on Antennas and Propagation, vol. 53, no. 11, pp. 3469-3476, 2005. 


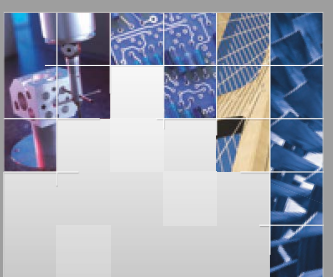

\section{Enfincering}
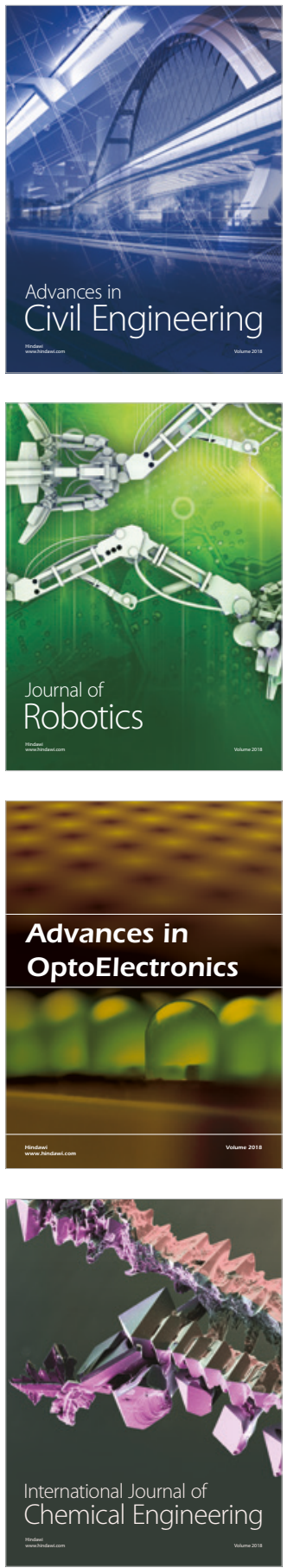

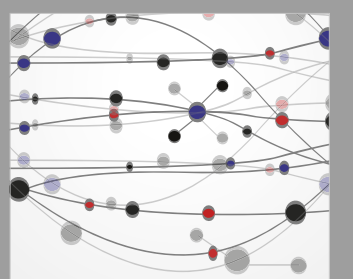

\section{Rotating \\ Machinery}

The Scientific World Journal

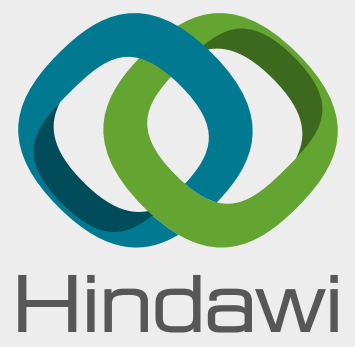

Submit your manuscripts at

www.hindawi.com
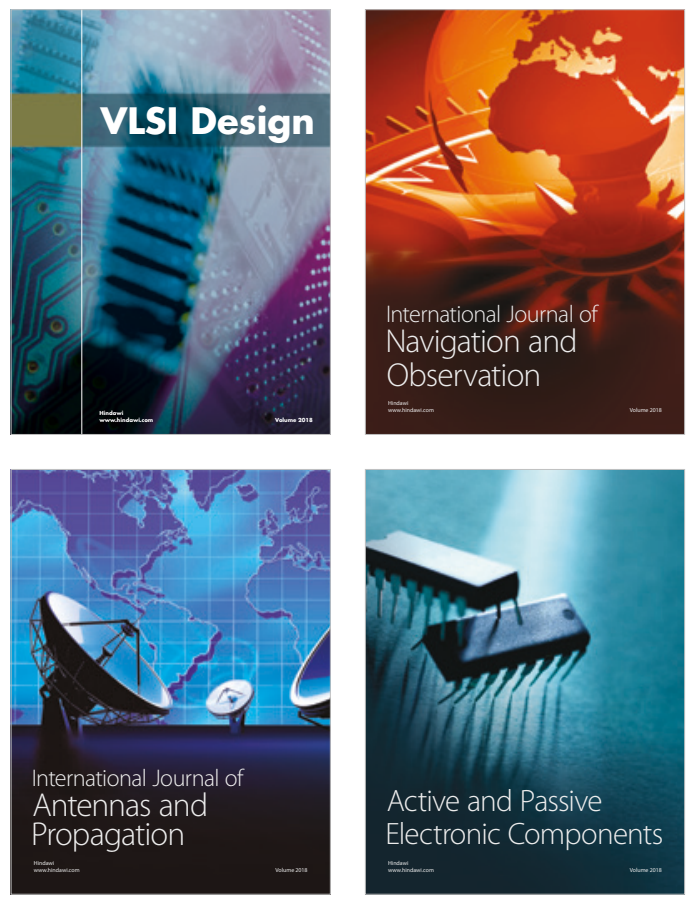
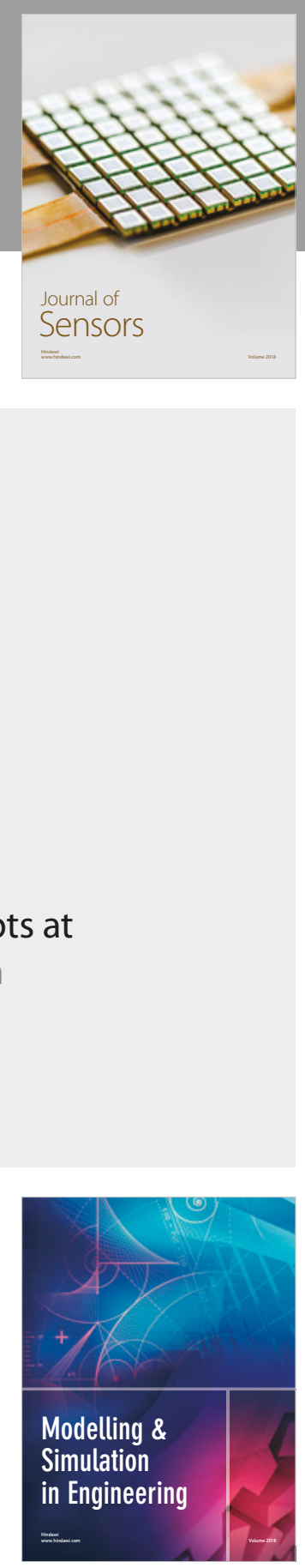

\section{Advances \\ Multimedia}
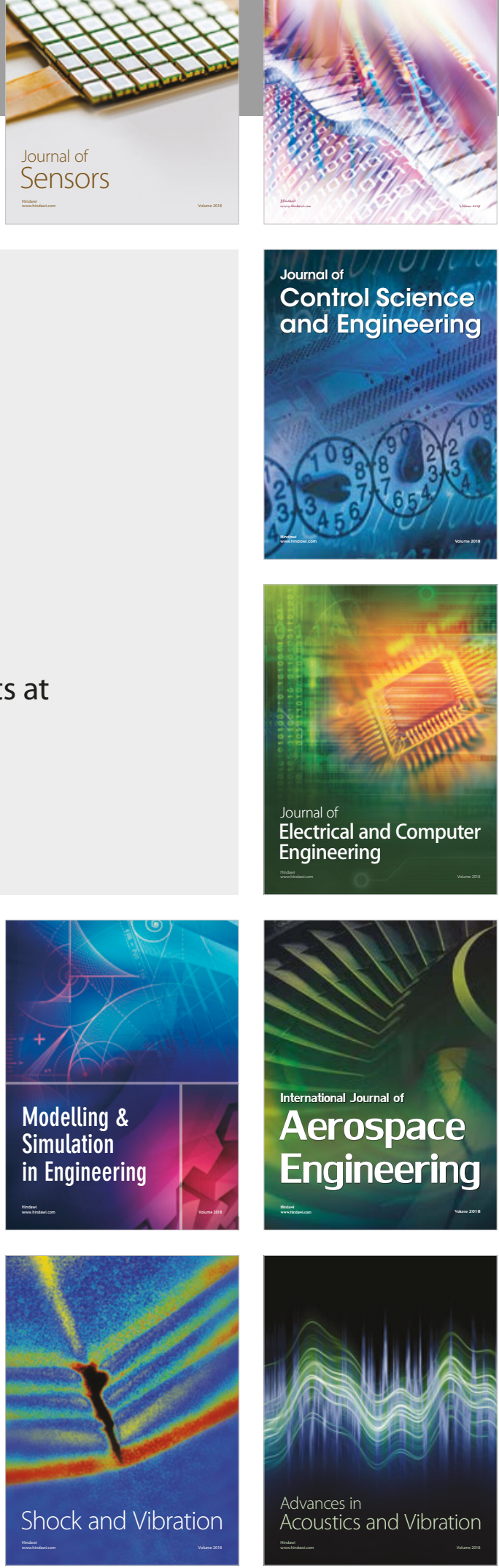